Incretin-based therapies and risk of pancreatic cancer in patients with type 2 diabetes : A meta-analysis of randomized controlled trials

\title{
Wang, Haining
}

2018-04

Wang , H , Liu , Y, Tian , Q , Yang , J , Lu , R , Zhan , S , Haukka , J \& Hong , T 2018 , ' Incretin-based therapies and risk of pancreatic cancer in patients with type 2 diabetes : A meta-analysis of randomized controlled trials ' , Diabetes, obesity and metabolism, vol. 20 , no. 4 , pp. $910-920$. https://doi.org/10.1111/dom. 13177

http://hdl.handle.net/10138/325808

https://doi.org/10.1111/dom.13177

unspecified

acceptedVersion

Downloaded from Helda, University of Helsinki institutional repository.

This is an electronic reprint of the original article.

This reprint may differ from the original in pagination and typographic detail.

Please cite the original version. 


\title{
[THIS IS NOT THE FINAL VERSION]
}

\section{Incretin-based therapies and risk of pancreatic cancer in patients with type 2} diabetes: a meta-analysis of randomised controlled trials

Short title: Incretin-based therapies and pancreatic cancer risk

\author{
Haining Wang, $\mathrm{MD}^{1^{*}} \mid$ Ye Liu, $\mathrm{MD}^{1^{*}} \mid$ Qing Tian, $\mathrm{MD}^{1} \mid$ Jin Yang, $\mathrm{MD}^{1}\left|\operatorname{Ran} \mathrm{Lu}, \mathrm{MD}^{1}\right|$ \\ Siyan Zhan, $\mathrm{PhD}^{2} \mid$ Jari Haukka, $\mathrm{PhD}^{3} \mid$ Tianpei Hong, $\mathrm{MD}, \mathrm{PhD}^{1}$
}

${ }^{1}$ Department of Endocrinology and Metabolism, Peking University Third Hospital, Beijing 100191,

China

${ }^{2}$ Department of Epidemiology and Biostatistics, School of Public Health, Peking University Health Science Center, Beijing 100191, China

${ }^{3}$ The Clinicum Department of Public Health, University of Helsinki, Helsinki, Finland

${ }^{*}$ These authors contributed equally to this work.

Corresponding author: Tianpei Hong, MD, PhD, Department of Endocrinology and Metabolism, Peking University Third Hospital, 49 North Garden Road, Haidian District, Beijing 100191, China. E-mail: tpho66@bjmu.edu.cn; Phone number: +86-10-82266918; Fax number: +86-10-62017700

Word count for abstract: 296

Word count for main manuscript: 3620 


\section{ABSTRACT}

2 Aims: Conflicting evidence exists regarding the potential risk of pancreatic cancer with use of incretin drugs in patients with type 2 diabetes (T2DM). We performed a meta-analysis of randomised controlled trials (RCTs), including six recently published large-scale cardiovascular outcome trials (CVOTs), to evaluate the risk of pancreatic cancer with incretin-based therapies in patients with T2DM.

Materials and methods: The PubMed, Embase, Cochrane Central Register and ClininalTrials.gov databases were searched for RCTs in T2DM that compared incretin drugs with placebo or other antidiabetic drugs, with treatment and follow-up durations of no less than 52 weeks, from January 1, 2007 to May 1, 2017. Two reviewers screened the studies, extracted the data and assessed the risk of bias independently and in duplicate.

Results: Thirty-three studies $(n=79,971)$, including the six CVOTs, with 87 pancreatic cancer events were identified. Overall, the pancreatic cancer risk was not increased in patients administered with incretin drugs compared to controls (Peto OR 0.67 [95\%CI 0.44 to 1.02$]$ ). In the six CVOTs, 79 pancreatic cancer events were identified in 55,248 subjects. Pooled estimates of the six CVOTs displayed the identical tendency (Peto OR 0.65 [95\%CI 0.42 to 1.01$]$ ). Notably, in the subgroup of patients who received treatment and follow-up for 104 weeks or more, 84 pancreatic cancer events were identified in 59,919 subjects, and a lower risk of pancreatic cancer was associated with incretin-based therapies (Peto OR 0.62 [95\%CI 0.41 to 0.95$]$ ).

Conclusions: Treatment with incretin drugs is not associated with an increased risk of pancreatic cancer in patients with T2DM. Instead, it might protect against the pancreatic malignancy in patients treated for 104 weeks or more. The major limitations of this study are that pancreatic safety was not the primary outcome of these enrolled trials, and the event number and follow-up time are limited.

KEYWORDS: incretins, GLP-1 analogue, dipeptidyl peptidase-4 inhibitors, type 2 diabetes, meta-analysis 


\section{1 | INTRODUCTION}

Incretin-based therapies include glucagon-like peptide-1 (GLP-1) receptor agonists and dipeptidyl peptidase4 (DPP-4) inhibitors. They demonstrate an anti-hyperglycaemic effect in a glucose-dependent manner and are beneficial for weight control. Recently, encouraging results from two cardiovascular outcome trials (CVOTs) have shown that GLP-1 receptor agonist can reduce the risk of major adverse cardiovascular events in patients with type 2 diabetes (T2DM) who are at high cardiovascular risk. ${ }^{1,2}$ Because of these favourable features, incretin drugs have been recommended as important therapeutic options for patients with T2DM. ${ }^{3}$

However, concerns have been raised for years about the pancreatic safety of incretin drugs. In 2011, Elashoff and colleagues ${ }^{4}$ reported that increased risks of both pancreatitis and pancreatic cancer were associated with the use of incretin drugs. Thereafter, attempts have been made to investigate the safety of incretin-based therapies. However, results from the preclinical reports ${ }^{5-7}$ and observational cohort studies ${ }^{8-11}$ are conflicting. Notably, the incidence of pancreatic cancer is low ${ }^{12}$. No individual trial has enough power to assess the risk of pancreatic malignancy sufficiently. Therefore, pooling data from large randomised controlled trials (RCTs) would be an alternative method of investigating this sa fety issue.

Recently, CVOTs of incretin drugs (e.g. EXAMINE, ${ }^{13}$ SAVOR, ${ }^{14} \mathrm{TECOS}^{15}{ }^{15}$ ELXA, ${ }^{16} \mathrm{LEADER}^{1}$ and SUSTAIN- $6^{2}$ ) have been completed or are ongoing. In these trials, a large number of patients were followed up for relatively longer periods and managed with a similar glycated haemoglobin $(\mathrm{HbA} 1 \mathrm{c})$ achievement goal. Accordingly, pooling data from these CVOTs might help researchers better understand the true risk of pancreatic malignancy with incretin-based therapies.

Here, we performed a meta-analysis of large RCTs, including the six recently published CVOTs, to evaluate the risk of pancreatic cancer with incretin-based therapies in patients with T2DM. 


\section{2 | MATERIALS AND METHODS}

2.1 | Data sources and searches

The PubMed, Embase and Cochrane Central Register databases were searched from January 1, 2007 to May 1, 2017 for RCTs that involved incretin drugs and were published in English. Medical subject headings and free text terms were used to identify the related articles. An endocrinologist, together with an epidemiologist, developed the search strategy (S1 Text, Supporting information). The ClinicalTrials.gov was searched using the same method to identify trials that were complete but unpublished. It also provided us with extended information about adverse events related with the selected trials. The search was performed on May 1, 2017.

\section{2 | Study selection}

We selected trials that satisfied the following criteria: 1) study type, RCTs; 2) subjects, adult patients with T2DM; 3) intervention and comparators, trials that compared the effects of incretin drugs (GLP-1 receptor agonists or DPP-4 inhibitors) with comparators (placebo or other antidiabetic drugs); 4) duration of treatment and followup, it is estimated that the mean time from first malignant cell to the clinical diagnosis of pancreatic cancer is 0.7 years in males and 0.6 years in females, ${ }^{17}$ therefore, we only included trials that had a treatment and follow-up time at least 52 weeks to reduce the bias related to the undiscovered pancreatic cancer before start of intervention; 5) sample size restriction, pancreatic cancer has a low incidence (10-14 per 100,000 person years), ${ }^{12}$ to reduce sampling variation, we only included the trials with at least 500 randomised subjects; and 6) outcome evaluation, the trials were required to have clear information of pancreatic malignancy, or at least systemic reports of neoplasms in the supplemental materials or in the data posted on ClinicalTrials.gov.

\section{3 | Data extraction and quality assessment}



evaluate the risk of bias and to collect data independently and in duplicate for each included trial. Disputes were discussed by the study group and were adjudicated by the study supervisor. For multiple reports of one trial, we only documented the data from the report with the longest follow-up. For each eligible trial included in this study, the characteristics of the trials, including National Clinical Trial (NCT) codes (if available), sample size, the number of participants in each treatment group, duration of treatment and follow-up, percentage of male participants, age and body mass index (BMI) of the participants, duration of diabetes and baseline HbAlc level were recorded. As glycaemic control status may affect the risk of cancer, ${ }^{18}$ we also recorded the final $\mathrm{HbA} \mathrm{c}$ difference between the groups. An HbAlc difference of more than $0.4 \%$ was regarded as clinically significant. ${ }^{19}$

The pancreatic cancer events in each group were recorded separately. The number of patients exposed to each treatment group was recorded using intention-to-treat (ITT) data.

A modified Cochrane Collaboration's tool, which includes information about the randomisation process, allocation concealment, blindness, adjudication of outcomes and selective reporting, was used to assess the risk of bias in each trial. ${ }^{20}$ We used funnel plot asymmetry to detect whether there was publication bias and Egger's regression test to measure funnel plot asymmetry in Stata $11.0 .^{21}$ 
of events. ${ }^{20}$ To determine the possible factors that might affect the risk of pancreatic cancer, we performed four prespecified subgroup analyses, according to the following stratifications: the duration of treatment and follow-up (52 to 103 weeks or no less than 104 weeks), class of incretin drugs (GLP-1 receptor agonists or DPP-4 inhibitors), type of comparators (placebo or other non-incretin antidiabetic drugs), and level of HbAlc difference between treatment arms at the end of trials (more than $0.4 \%$ or not). In addition, a sensitivity analysis was performed using alternative effect measures (OR vs. relative risk), pooling methods (Peto method vs. Mantel-Haenszel method), and consideration of heterogeneity (fixed effect $v s$. random effect). We reported our results, according to the PRISMA statement. ${ }^{22}$

\section{3 | RESULTS}

Among the 5,416 potential reports from PubMed, Embase and Cochrane Central Register, and 305 reports from ClinicalTrial.gov, we identified 622 reports for full-text reviews. Finally, thirty-three RCTs, , $2,13-16,23-49$ including the six recently reported CVOTs, ${ }^{1,2,13-16}$ fulfilled the inclusion criteria (32 from published journals and 1 unpublished trial from ClinicalTrials.gov). A flow diagram of the trial selection is presented in Figure 1. For all included studies, the average age of the participants ranged from 51.8 to 72.6 years, and the mean BMI ranged from 24.9 to $37.1 \mathrm{~kg} / \mathrm{m}^{2}$, with a mean duration of diabetes ranging from 1.0 to 13.9 years. The percentage of male subjects ranged from $43 \%$ to $71 \%$. The average baseline HbAlc level ranged from $7.2 \%$ to $9.2 \%$. The mean or median follow-up time ranged from 52 to 198 weeks (Table 1).

\section{$3.1 \mid$ Quality of the included trials and publication bias}

Among the included trials in our analysis, randomisation was well designed in 31 studies. One trial did not mention how the random sequence was generated. One trial was at high risk of bias because its randomisation was stratified by different baseline treatments. For allocation concealment and blinding of the treatment, six trials 
without treatment concealment to the investigators and participants were regarded as having a high risk of bias.

As for the outcome evaluation, all included studies provided safety data for the ITT population, and the six CVOTs and nine non-CVOT studies had an independent adjudication committee for the cancer and pancreatitis events, which were at low risk of bias (Table S1, Supporting information).

The funnel plot was symmetric (Egger's test $P=0.887$ ) (Figure S1, Supporting information). Moreover, all included trials were designed to test the drug's efficacy of glucose lowering or the safety of cardiovascular outcomes. Therefore, pancreatic cancer and pancreatitis events had a minor effect on the selected publications.

\section{2 | Risk of pancreatic cancer in the pooled analysis}

Of the 33 included RCTs, eleven studies reported pancreatic cancer events. Thirty-five events were reported in 42,233 incretin group subjects, and 52 events were reported in 37,738 control group subjects. Pooled estimates of the 33 trials $(n=79,971)$ showed that no increased risk of pancreatic cancer was associated with the incretin drugs compared to the controls (Peto OR 0.67 [95\%CI 0.44 to 1.02]). In particular, in the six CVOTs, thirty-three events were reported in 27,663 subjects in the incretin group, and 48 events were reported in 27,585 control group subjects. The Peto OR of the pooled analysis of the six CVOTs $(n=55,248)$ was 0.65 [95\%CI 0.42 to 1.01$]$ (Figure 2).

\subsection{Risk of pancreatic cancer in the subgroup analysis}

When evaluating the effect of incretin drugs on the risk of pancreatic cancer, the exposure time is an important factor. Among the trials that followed subjects for 52-103 weeks, three pancreatic cancer events in 11,765 incretin users and no events in 8,287 non-incretin users were observed (Peto OR 5.63 [95\%CI 0.52 to 60.4]) (Figure S2, Supporting information). For the subjects who received incretin treatment and were followed for 104 weeks or 
more, the risk of pancreatic cancer decreased significantly, compared to subjects who received the control treatment (Peto OR 0.62 [95\%CI 0.41 to 0.95]) (Figure 3).

No significant difference in the risk of pancreatic cancer was observed in the subgroup analysis of the class of incretin drugs (Peto OR 0.77 [95\%CI 0.42 to 1.42 ] for GLP-1 receptor agonists and 0.59 [95\% CI 0.33 to 1.05 ] for DPP4 inhibitors). In the subgroup analysis of the type of comparators, we did not find any significant difference in the risk of pancreatic cancer in the subgroups of incretins vs. active controls (Peto OR 1.12 [95\%CI 0.25 to 5.06]), such as metformin, thiazolidinediones, sulfonylureas, insulin and sodium-dependent glucose transporters 2 inhibitors. A decreased risk of pancreatic cancer was observed in the incretin group compared to the placebo group (Peto OR 0.63 [95\%CI 0.40 to 0.97$]$ ). In addition, there was no significant difference in the risk of pancreatic cancer between the incretin-based and control therapies in the subgroup stratified by the level of the final $\mathrm{HbAlc}$ difference (Peto OR 0.70 [95\%CI 0.45 to 1.09 ] and 0.45 [95\%CI 0.12 to 1.70 ] for the subgroups with $\mathrm{HbA} 1 \mathrm{c}$ differences $\leq 0.4 \%$ and $>0.4 \%$, respectively) (FigureS3-5, Supporting information).

\section{4 | Risk of pancreatitis in the pooled analysis}

The overall pancreatitis risk was not increased in the incretin group compared with the control group (Peto OR 1.12 [95\%CI 0.85 to 1.47$]$ ). Pooled analysis of the six CVOTs did not show an increased risk of pancreatitis associated with incretin-based therapies (Peto OR 1.06 [95\% CI 0.80 to 1.42]) (Figure S6, Supporting information).

\section{5 | Sensitivity analysis}

The sensitivity analysis of pancreatic cancer risk using an alternative pooling method (Mantel-Haenszel OR 0.67 [ $95 \% \mathrm{CI} 0.44$ to 1.02$]$ ), effect measure (relative risk 0.67 [ $95 \% \mathrm{CI} 0.44$ to 1.02$]$ ), and consideration of 
heterogeneity (random effects OR 0.68 [95\%CI 0.44 to 1.06$]$ ) did not show any important change in the pooled effects.

\section{4 | DISCUSSION}

Overall, we screened 5,721 studies and included 33 eligible RCTs reporting 87 pancreatic cancer events among 79,971 patients. We found that compared with the controls, treatment with incretin drugs was not associated with an increased risk of pancreatic cancer in patients with T2DM. Instead, use of incretin drugs for 104 weeks or more might even decrease the risk of pancreatic malignancy by $38 \%$ compared with controls.

The association between incretin-based therapies and pancreatic cancer has drawn a great concern recently. Unfortunately, neither preclinical studies ${ }^{5-7,50}$ nor the following cohort studies have answered this question consistently. ${ }^{8-11,51,52}$ In a recent large multinational cohort study, the risk of pancreatic cancer even seemed to be lower with longer incretin-based therapy durations (HR 1.53 [95\% CI 0.93 to 2.51 ], 1.07 [95\% 0.82 to 1.39 ] and 0.62 [ $95 \%$ CI 0.36 to 1.07 ] for duration of use $<1$ year, $1-1.9$ years and $\geq 2$ years, respectively), although the difference was not statistically significant. ${ }^{8}$ Additionally, the United Kingdom clinical practice research datalink (UK-CPRD) cohort study has reported that the minor increase of pancreatic cancer risk in new incretin users [adjusted HR 1.67 (1.01-2.77)] was likely caused by protopathic bias because of the lack of a duration of use and dosage effect for incretin agents on pancreatic cancer risk. ${ }^{11}$ Inconsistency and methodological limitations undermined the strength of those results. In the cohort studies, baseline characteristics and metabolic control levels could not be well matched between groups. Even in the nested case-control study, the incretin group still differed from the control group in the parameters that could affect the incidence of malignancy, including age, duration of diabetes, BMI and HbA1c levels. ${ }^{8}$ Furthermore, in most cohort studies, the report of pancreatic cancer events was based on medical or insurance records, which may have led to the inaccurate definition of the events. 

two meta-analyses by Monami and colleagues have suggested that there is no increased risk of pancreatic cancer

associated with the use of incretins. ${ }^{53,54}$ In the 2014 report, the primary outcome was pancreatitis, and the data collection was not based on pancreatic cancer. ${ }^{53}$ In addition, the sample size of each trial varied greatly (from 24 to 9340). The follow-up durations of the enrolled trials were not long enough (more than $70 \%$ of the trials had follow-up durations of 12-51 weeks), ${ }^{53,54}$ which did not take the latent period of cancer into consideration.

In our study, we enrolled qualified RCTs with baseline characteristics that were balanced between the groups. The drug exposure and follow-up were clear and well managed. All patients treated with incretin drugs were new users, thereby avoiding the bias in cohort studies caused by combining new users with prevalent users and the possible protopathic bias ${ }^{11}$. Note that pancreatic cancer is insidious and rare. The estimated time from first malignant cell to the clinical diagnosis of pancreatic cancer is $0.6-0.7$ year. ${ }^{17}$ Therefore, we only included trials with treatment and follow-up durations of at least 52 weeks to reduce the possibility of occult pancreatic malignancy at the start of trials, and we excluded studies with fewer than 500 subjects in case that cancer events reported in small trials by chance could dramatically affect the incidence. Recently, several large-scale CVOTs of incretin drugs have been completed or are ongoing. A large number of patients (from 3,927 to 16,492) were enrolled, and the duration of follow-up was much longer (median duration ranged from 1.5 to 3 years) in these trials. Moreover, the primary endpoint of these CVOTs was drug safety rather than efficacy, and patients in different intervention groups were managed under a similar glycaemic goal. Therefore, the differences in the HbAlc level achieved between the incretin and control groups were relatively small, providing a more parallel metabolic status. Accordingly, pooling data from these CVOTs might help us to better understand the pancreatic safety issue of long-term incretin-based therapies. ${ }^{55-57}$ Nauck and colleagues have remarked that CVOT studies 
could provide us with valuable information about pancreatic safeness, and they provided good evidence against previous estimates of the increased pancreatic cancer risk. ${ }^{57}$ We collected all available RCTs with follow-up times of at least 52 weeks, thus, our results could further support their conclusion.

It is known that patients with T2DM are at high risk of developing pancreatic cancer, with high mortality rates. ${ }^{58}$ Unfortunately, pancreatic cancer still lacks an effective management strategy and even presents with increasing incidence and mortality. ${ }^{12}$ Here, our subgroup analysis showed that treatment with incretin drugs for 2 years or more significantly reduced the risk of pancreatic cancer, compared to the controls, by $38 \%$. Notably, a similar trend was also observed in the large nested case-control cohort study mentioned above (HR 0.62 [95\%CI 0.36 to 1.07$]){ }^{8}$ Signals from laboratory studies have also suggested an anti-tumour effect of incretin drugs. In our previous studies, we found that GLP-1 receptor levels were lower in cancer tissues than in tumour adjacent pancreatic tissues, and a lower GLP-1 receptor level was associated with poorer prognoses in patients with pancreatic cancer. Moreover, GLP-1 receptor activation with liraglutide inhibited growth and promoted apoptosis of human pancreatic cancer cells in a GLP-1 receptor-dependent manner in vitro, and attenuated pancreatic tumour growth in a mouse xenograft model in vivo. ${ }^{59,60}$ In agreement with our previous findings, it has been shown that GLP-1 receptor agonist exendin-4 can inhibit cell growth in colon cancer cells ${ }^{61}$ and breast cancer cells ${ }^{62,63}$ in vitro and in vivo. Furthermore, exendin-4 also counteracts the invasive potential of human neuroblastoma cells. ${ }^{64}$ These $^{-1}$ consistent signals from clinical and laboratory studies suggest that long-term incretin-based therapies might shed some light on how to prevent the development and progression of pancreatic malignancy, which usually has a poor outcome.

GLP-1 receptor agonists and DPP-4 inhibitors are both incretin drugs, however, they act differently. GLP-1 receptor agonists directly and intensively stimulate GLP-1 receptor and its downstream signalling pathways, while DPP-4 inhibitors can increase the levels of endogenous incretin hormones by inhibiting DPP-4-mediated incretin 
degradation. Hence, it has been suggested to analyse them separately. ${ }^{56}$ Here, we found no difference between GLP-1 receptor agonists and DPP-4 inhibitors, in terms of their association with pancreatic cancer risk. It is well known that diabetes itself is an independent risk factor for pancreatic cancer and its high mortality. ${ }^{12,58}$ Although patients had parallel baseline $\mathrm{HbAlc}$ levels when they entered a trial, they received different antidiabetic therapies and might significantly vary in their glycaemic control. Therefore, we conducted a prespecified subgroup analysis based on a $0.4 \%$ difference in the final $\mathrm{HbAl}$ c level, which is usually considered to be the non-inferiority margin, ${ }^{19}$ to clarify whether our result would be altered by glycaemic control variations. Again, we could not find any increased risk of pancreatic cancer associated with incretin drugs in the subgroups stratified by level of HbAlc difference, suggesting the consistency of the observations that incretin drugs were not the promoter of pancreatic cancer.

It has been argued that incretin drugs were a potential inducer of acute and chronic pancreatitis, ${ }^{65-67}$ thus in the long run promoting the development of pre-neoplastic lesions and increasing the risk of pancreatic cancer However, increasing reports from clinical trials,${ }^{68}$ cohort studies $^{69,70}$ and systemic reviews ${ }^{53,71}$ have shown no increased risk of pancreatitis associated with incretin-based therapies. Here, we also found results similar to those reports, providing additional evidence for the pancreatic safety of incretin-based therapies.

Several limitations should be considered in our study. First, pancreatic safety was not the primary outcome of the included trials, and the number of pancreatic cancer events in our study was relatively smaller than that in some observational cohort studies. ${ }^{8,9,11}$ This limitation is primarily attributed to the nature of RCTs because it is not practical for a RCT to enrol such a large population as the cohort studies performed using databases. Nevertheless, signals from our study and most cohort studies consistently suggest that incretin drugs were not the carcinogen of pancreatic malignancy. Second, we noticed that $91 \%$ of pancreatic cancer events were reported in the six CVOTs, whereas the number of the events was small ( 8 cases in 24,723 subjects) which led to a wide range 
of $95 \% \mathrm{CI}$ in the non-CVOT studies. There might be underreporting in some of the non-CVOTs with no pancreatic malignancies reported. However, the nature of RCTs could partially balance the possibility of underreporting. Moreover, the total number of pancreatic cancer events in our pooling data was 87 in 79,971 subjects (approximately 108 per 100,000 persons). The estimated overall incidence was approximately 47 per 100,000 person years in all trials and 55 per 100,000 person years in trials with follow-ups of 104 weeks or more, which was much higher than that in the general population (10-14 per 100,000 person years $)^{12}$ and similar to that reported in a previous large cohort study in patients with T2DM (60 per 100,000 person years). ${ }^{8}$ Therefore, there might be no obvious underreporting in the trials included in our study, and the limited number of the cancer events may not substantially undermine our results. Third, we did not have the primary time-to-event data for all included trials, and it is possible that a risk of immortal time bias might exist in our subgroup analysis. However, in the nested case-control cohort study, the declined tendency of pancreatic cancer risk was also found among the subjects treated with incretin drugs for 2 years or more. ${ }^{8}$ Furthermore, incretins are not carcinogens, and they may influence the rate of neoplasm progression and affect the time period from the first malignant cell to the clinical diagnosis of pancreatic cancer. Theoretically, the longer the exposure, the more significant effect could be found. Therefore, pooling data from more large-scale trials with long-term incretin-based therapies, particularly the CVOTs with the primary time-to-event data, might provide us with a clearer picture on this topic. Fourth, we did not identify pancreatitis as acute or chronic, partly because some trials did not define or report pancreatitis in detail. After all, it was not the primary outcome in this analysis.

In conclusion, our meta-analysis of 33 RCTs involving 79,971 subjects suggests that treatment with incretin drugs for no less than 52 weeks is not associated with an increased risk of pancreatic cancer in patients with T2DM. Instead, treatment with incretin drugs might protect against the risk of pancreatic malignancy, particularly in 
patients with T2DM who received the treatment for 104 weeks or more. Even so, it is difficult to verify this issue

in a single study because pancreatic cancer is rare and occult. Accordingly, pooling more data from large-scale RCTs, particularly long-term CVOTs, may help us to find the true answer to the question of whether incretinbased therapies are safe and might even protect patients with T2DM against pancreatic malignancy.

Figure and table legends.

Figure 1. Flow diagram of trial selection

Table 1. Characteristics of randomised controlled trials of incretin-based therapies and pancreatic cancer events in patients with type 2 diabetes

NCT, National Clinical Trial. NR, not reported. * Final HbAlc (\%) difference: incretin group vs. control group at the end of the trial. ${ }^{\dagger}$ There was no publication of the studies, and presented here is the time of the last data update on ClinicalTrial.gov website. ${ }^{\S}$ Sitagliptin/metformin fixed-dose combination. ${ }^{\star}$ No report of pancreatic cancer in the article, but there were systemic reports of neoplasms in the supplemental materials or in the data posted on the ClinicalTrial.gov website.

Figure 2. Risk of pancreatic cancer in patients with type 2 diabetes who were treated with incretin drugs or controls.

CVOT, cardiovascular outcome trial.

Figure 3. Risk of pancreatic cancer in patients with type 2 diabetes who were treated with incretin drugs or controls and followed up for 104 weeks or more.

Acknowledgments: We thank Ph.D. candidate Jun Yang from the Department of Epidemiology and Biostatistics,

School of Public Health, Peking University Health Science Center, for his help with the literature screening.

Competing interests: T.H. has served on advisory boards for Novo Nordisk, Eli Lilly and Company, Sanofi, 
any other potential conflicts of interest relevant to this article.

\section{Author Contributions:}

T.H. was involved in designing the meta-analysis and data collection and analysis, as well as the writing, editing and revising of the manuscript. H.W and Y.L were involved in the data collection and analysis, as well as writing the manuscript. Q.T, J.Y, R.L were involved in the data collection, quality evaluation of the trials and manuscript editing. Z.S and J.H were involved in the study methodology and data analysis, as well as reviewing and editing the manuscript. All authors reviewed the manuscript, approved the final draft and agreed to submit it for publication.

Funding: This study was partly funded by the National Natural Sciences Foundation of China (81570692, 81670701, 81401142, and 81400767). The funders played no role in the study design, data collection and analysis, decision to publish, or preparation of the manuscript.

\section{References}

1. Marso SP, Daniels GH, Brown-Frandsen K, et al. Liraglutide and cardiovascular outcomes in type 2 diabetes. N Engl J Med. 2016;375: 311-22. doi:10.1056/NEJMoa1603827. PMID: 27295427

2. Marso SP, Bain SC, Consoli A, et al. Semaglutide and cardiovascular outcomes in patients with type 2 diabetes. N Engl J Med. 2016;375: 1834-44. doi:10.1056/NEJMoa1607141. PMID: 27633186

3. American Diabetes Association. Standards of Medical Care in Diabetes. Diabetes Care. 2017;40: S1-S135. [cited 2017May20]. Available from: http://care.diabetesjournals.org on 9 June 2017. 
4. Elashoff M, Matveyenko AV, Gier B, Elashoff R, Butler PC. Pancreatitis, pancreatic, and thyroid cancer with glucagon-like peptide-1-based therapies. Gastroenterology. 2011;141: 150-6. doi:10.1053/j.gastro.2011.02.018. PMID: 21334333

5. Butler AE, Campbell-Thompson M, Gurlo T, Dawson DW, Atkinson M, Butler PC. Marked expansion of exocrine and endocrine pancreas with incretin therapy in humans with increased exocrine pancreas dysplasia and the potential for glucagon-producing neuroendocrine tumors. Diabetes. 2013;62: 2595-604. doi:10.2337/db12-1686. PMID: 23524641

6. Nyborg NC, Mølck AM, Madsen LW, Knudsen LB. The human GLP-1 analog liraglutide and the pancreas: evidence for the absence of structural pancreatic changes in three species. Diabetes. 2012;61: 1243-9. doi:10.2337/db11-0936. PMID: 22338093

7. Gotfredsen CF, Mølck AM, Thorup I, et al. The human GLP-1 analogs liraglutide and semaglutide: absence of histopathological effects on the pancreas in nonhuman primates. Diabetes. 2014;63: $2486-97$. doi:10.2337/db13-1087. PMID: 24608440

8. Azoulay L, Filion KB, Platt RW, et al. Incretin based drugs and the risk of pancreatic cancer: international multicentre cohort study. BMJ. 2016;352:i581. doi:10.1136/bmj.i581. PMID: 26888382

9. Gokhale M, Buse JB, Gray CL, Pate V, Marquis MA, Stürmer T. Dipeptidyl-peptidase-4 inhibitors and pancreatic cancer: a cohort study. Diabetes Obes Metab. 2014;16: 1247-56. doi:10.1111/dom.12379. PMID: 25109825

10. Tseng CH. Sitagliptin and pancreatic cancer risk in patients with type 2 diabetes. Eur J Clin Invest. 2016;46: 70-9. doi:10.1111/eci.12570. PMID: 26584246

11. Knapen LM, van Dalem J, Keulemans YC, van Erp NP, Bazelier MT, De Bruin ML, Leufkens HGM, Croes S, Neef C, de Vries F, Driessen JHM. Use of incretin agents and risk of pancreatic cancer: a population-based 
cohort study. Diabetes Obes Metab. 2016;18(3):258-265. doi:10.1111/dom.12605. PMID: 26537555

12. Ryerson AB, Eheman CR, Altekruse SF, et al. Annual Report to the Nation on the Status of Cancer, 19752012, featuring the increasing incidence of liver cancer. Cancer. 2016;122: 1312-37. doi:10.1002/cncr.29936. PMID: 26959385

13. White WB, Cannon CP, Heller SR, et al. Alogliptin after acute coronary syndrome in patients with type 2 diabetes. $N$ Engl J Med. 2013;369: 1327-35. doi:10.1056/NEJMoa1305889. PMID: 23992602

14. Scirica BM, Bhatt DL, Braunwald E, et al. Saxagliptin and cardiovascular outcomes in patients with type 2 diabetes mellitus. $N$ Engl J Med. 2013;369: 1317-26. doi:10.1056/NEJMoa1307684. PMID: 23992601

15. Green JB, Bethel MA, Armstrong PW, et al. Effect of sitagliptin on cardiovascular outcomes in type 2 diabetes. NEngl J Med. 2015;373: 232-42. doi:10.1056/NEJMoa1501352. PMID: 26052984

16. Pfeffer MA, Claggett B, Diaz R, et al. Lixisenatide in patients with type 2 diabetes and acute coronary syndrome. N Engl J Med. 2015;373: 2247-57. doi:10.1056/NEJMoa1509225. PMID: 26630143

17. Luebeck EG, Curtius K, Jeon J, Hazelton WD. Impact of tumor progression on cancer incidence curves. Cancer Res. 2013;73: 1086-96. doi: 10.1158/0008-5472.CAN-12-2198. PMID: 23054397

18. Chang SC, Yang WV.Hyperglycemia, tumorigenesis, and chronic inflammation. Crit Rev Oncol Hematol. 2016;108: 146-153. doi:10.1016/j.critrevonc.2016.11.003. PMID: 27931833

19. The US Center for Drug Evaluation and Research Food and Drug Administration. Guidance for Industry ucm071624. [cited 2017 Oct 3] Available from: https://www.fda.gov/downloads/Drugs/Guidances/ ucm071624.pdf.

20. Jonathan JD, Julian PTH, Douglas GA. Analysing data and undertaking meta-analyses. In: Julian H, Sally G, editors. Cochrane handbook for systematic reviews of interventions. Version 5.1.0, 2011. [cited 2017 May20]. Available from:www.cochrane.org/resources/handbook. 
21. Sterne JA, Egger M, Smith GD. Systematic reviews in health care: Investigating and dealing with publication and other biases in meta-analysis. BMJ. 2001;323: 101-5. PMID: 11451790

22. Moher D, Liberati A, Tetzlaff J, Altman DG. Preferred reporting items for systematic reviews and metaanalyses: the PRISMA statement. BMJ. 2009;339:b2535. doi:10.1136/bmj.b2535. PMID: 19622551

23. Company ELA. A Study Comparing the Effects and Safety of Dulaglutide With Insulin Glargine in Type 2 Diabetes Mellitus: National Library of Medicine (US), 2000. [cited 2017 May 1]. Available from: https://www.clinicaltrials.gov/ct2/show/NCT01648582

24. Lewin A, DeFronzo RA, Patel S, et al. Initial combination of empagliflozin and linagliptin in subjects with type 2 diabetes. Diabetes Care. 2015;38: 394-402. doi:10.2337/dc14-2365. PMID: 25633662

25. Gough SC, Bode BW, Woo VC, et al. One-year efficacy and safety of a fixed combination of insulin degludec and liraglutide in patients with type 2 diabetes: results of a 26 -week extension to a 26-week main trial. Diabetes Obes Metab. 2015;17: 965-73.doi:10.1111/dom.12498. PMID: 25980900

26. Roden M, Merker L, Christiansen AV, et al. Safety, tolerability and effects on cardiometabolic risk factors of empagliflozin monotherapy in drug-naive patients with type 2 diabetes: a double-blind extension of a Phase III randomized controlled trial. Cardiovasc Diabetol. 2015;14: 154. doi:10.1186/s12933-015-0314-0. PMID: 26701110

27. Davies MJ, Bergenstal R, Bode B, et al. Efficacy of liraglutide for weight loss among patients with type 2 diabetes: The SCALE diabetes randomized clinical trial. JAMA. 2015;314: 687-99. doi:10.1001/jama.2015.9676. PMID: 26284720

28. Blonde L, Jendle J, Gross J, et al. Once-weekly dulaglutide versus bedtime insulin glargine, both in combination with prandial insulin lispro, in patients with type 2 diabetes (AWARD-4): a randomised, openlabel, phase 3, non-inferiority study. Lancet. 2015;385: 2057-66. doi:10.1016/S0140-6736(15)60936-9. PMID: 
29. Giorgino F, Benroubi M, Sun JH, Zimmermann AG, Pechtner V. Efficacy and safety of once-weekly dulaglutide versus insulin glargine in patients with type 2 diabetes on metformin and glimepiride (AWARD-

2). Diabetes Care. 2015;38: 2241-9. doi:10.2337/dc14-1625. PMID: 26089386

30. Schernthaner G, Durán-Garcia S, Hanefeld M, et al. Efficacy and tolerability of saxagliptin compared with glimepiride in elderly patients with type 2 diabetes: a randomized, controlled study (GENERATION). Diabetes Obes Metab. 2015;17: 630-8. doi:10.1111/dom.12461. PMID: 25761977

31. Home PD, Shamanna P, Stewart M, et al. Efficacy and tolerability of albiglutide versus placebo or pioglitazone over 1 year in people with type 2 diabetes currently taking metformin and glimepiride: HARMONY 5. Diabetes Obes Metab. 2015;17: 179-87. doi:10.1111/dom.12414. PMID: 25406730

32. Umpierrez G, Tofé PS, PérezMF, Shurzinske L, Pechtner V. Efficacy and safety of dulaglutide monotherapy versus metformin in type 2 diabetes in a randomized controlled trial (AWARD-3). Diabetes Care. 2014;37: 2168-76. doi:10.2337/dc13-2759. PMID: 24842985

33. Rosenstock J, Fonseca VA, Gross JL, et al. Advancing basal insulin replacement in type 2 diabetes inadequately controlled with insulin glargine plus oral agents: a comparison of adding albiglutide, a weekly GLP-1 receptor agonist, versus thrice-daily prandial insulin lispro. Diabetes Care. 2014;37: 2317-25. doi:10.2337/dc14-0001. PMID: 24898300

34. Weissman PN, Carr MC, Ye J, et al. HARMONY 4: randomised clinical trial comparing once-weekly albiglutide and insulin glargine in patients with type 2 diabetes inadequately controlled with metfor min with or without sulfonylurea. Diabetologia. 2014;57: 2475-84. doi:10.1007/s00125-014-3360-3. PMID: 25208756

35. Ahren B, Johnson SL, Stewart M, et al. HARMONY 3: 104-week randomized, double-blind, placebo- and active-controlled trial assessing the efficacy and safety of albiglutide compared with placebo, sitagliptin, and 
glimepiride in patients with type 2 diabetes taking metformin. Diabetes Care. 2014;37: 2141-8. doi:10.2337/dc14-0024. PMID: 24898304

36. Henry RR, Staels B, Fonseca VA, et al. Efficacy and safety of initial combination treatment with sitagliptin and pioglitazone--a factorial study. Diabetes Obes Metab. 2014;16: 223-30. doi:10.1111/dom.12194. PMID: 23909985

37. Inagaki N, Watada $\mathrm{H}$, Murai $\mathrm{M}$, et al. Linagliptin provides effective, well-tolerated add-on therapy to preexisting oral antidiabetic therapy over 1 year in Japanese patients with type 2 diabetes. Diabetes Obes Metab. 2013;15: 833-43. doi:10.1111/dom.12110. PMID: 23565760

38. Yki-Järvinen H, Rosenstock J, Durán-Garcia S, et al. Effects of adding linagliptin to basal insulin regimen for inadequately controlled type 2 diabetes: a $\geq 52$-week randomized, double-blind study. Diabetes Care. 2013;36: 3875-81. doi:10.2337/dc12-2718. PMID: 24062327

39. Haak T, Meinicke T, Jones R, Weber S, von Eynatten M, Woerle HJ. Initial combination of linagliptin and metformin in patients with type 2 diabetes: efficacy and safety in a randomised, double-blind 1-year extension study. Int J Clin Pract. 2013;67: 1283-93. doi:10.1111/ijcp.12308. PMID: 24118640

40. Göke B, Gallwitz B, Eriksson JG, Hellqvist Å, Gause-Nilsson I. Saxagliptin vs. glipizide as add-on therapy in patients with type 2 diabetes mellitus inadequately controlled on metformin alone: long-term (52-week) extension of a 52-week randomised controlled trial. Int J Clin Pract. 2013;67: 307-16. PMID: 23638466

41. Nauck M, Frid A, Hermansen K, et al. Long-term efficacy and safety comparison of liraglutide, glimepiride and placebo, all in combination with metformin in type 2 diabetes: 2 -year results from the LEAD-2 study. Diabetes Obes Metab. 2013;15: 204-12. doi:10.1111/dom.12012. PMID: 22985213

42. Gallwitz B, Rosenstock J, Rauch T, et al. 2-year efficacy and safety of linagliptin compared with glimepiride in patients with type 2 diabetes inadequately controlled on metformin: a randomised, double-blind, non- 
inferiority trial. Lancet. 2012;380: 475-83. doi:10.1016/S0140-6736(12)60691-6. PMID: 22748821

43. Gallwitz B, Guzman J, Dotta F, et al. Exenatide twice daily versus glimepiride for prevention of glycaemic deterioration in patients with type 2 diabetes with metformin failure (EUREXA): an open-label, randomised controlled trial. Lancet. 2012;379: 2270-8. doi:10.1016/S0140-6736(12)60479-6. PMID: 22683137

44. Chacra AR, Tan GH, Ravichandran S, List J, Chen R. Safety and efficacy of saxagliptin in combination with submaximal sulphonylurea versus up-titrated sulphonylurea over 76 weeks. Diab Vasc Dis Res. 2011;8: 1509. doi:10.1177/1479164111404574. PMID: 21562067

45. Hollander PL, Li J, Frederich R, Allen E, Chen R.Safety and efficacy of saxagliptin added to thiazolidinedione over 76 weeks in patients with type 2 diabetes mellitus. Diab Vasc Dis Res. 2011;8: 125-35. doi:10.1177/1479164111404575. PMID: 21562064

46. Seck T, Nauck M, Sheng D, et al. Safety and efficacy of treatment with sitagliptin or glipizide in patients with type 2 diabetes inadequately controlled on metformin: a 2-year study. Int J Clin Pract. 2010;64: 562-76. doi:10.1111/j.1742-1241.2010.02353.x. PMID: 20456211

47. Garber A, Henry R, Ratner R, et al. Liraglutide versus glimepiride monotherapy for type 2 diabetes (LEAD-3 Mono): a randomised, 52-week, phase III, double-blind, parallel-treatment trial. Lancet. 2009;373: 473-81. doi:10.1016/S0140-6736(08)61246-5. PMID: 18819705

48. Goldstein BJ, Feinglos MN, Lunceford JK, Johnson J, Williams-Herman DE. Effect of initial combination therapy with sitagliptin, a dipeptidyl peptidase-4 inhibitor, and metformin on glycemic control in patients with type 2 diabetes. Diabetes Care. 2007;30: 1979-87. doi:10.2337/dc07-0627. PMID: 17485570

49. Nauck MA, Duran S, Kim D, et al. A comparison of twice-daily exenatide and biphasic insulin aspart in patients with type 2 diabetes who were suboptimally controlled with sulfonylurea and metformin: a noninferiority study. Diabetologia. 2007;50: 259-67. doi:10.1007/s00125-006-0510-2. PMID: 17160407 
50. Aston-Mourney K, Subramanian SL, Zraika S, et al. One year of sitagliptin treatment protects against islet amyloid-associated beta-cell loss and does not induce pancreatitis or pancreatic neoplasia in mice. Am J Physiol Endocrinol Metab. 2013;305: E475-84. doi:10.1152/ajpendo.00025.2013. PMID: 23736544

51. Romley JA, Goldman DP, Solomon M, McFadden D, Peters AL. Exenatide therapy and the risk of pancreatitis and pancreatic cancer in a privately insured population. Diabetes Technol Ther. 2012;14: 904-11. doi:10.1089/dia.2012.0075. PMID: 22845701

52. Dore DD, Seeger JD, Chan KA. Incidence of health insurance claims for thyroid neoplasm and pancreatic malignancy in association with exenatide: signal refinement using active safety surveillance. Ther Adv Drug Saf. 2012;3: 157-64. doi:10.1177/2042098612446473. PMID: 25083233

53. Monami M, Dicembrini I, Mannucci E. Dipeptidyl peptidase-4 inhibitors and pancreatitis risk: a meta-analysis of randomized clinical trials. Diabetes Obes Metab. 2014;16: 48-56. doi:10.1111/dom.12176. PMID: 23837679

54.Monami M, Nreu B, Scatena A, et al. Safety issues with glucagon-like peptide-1 receptor agonists (pancreatitis, pancreatic cancer and cholelithiasis): Data from randomized controlled trials.Diabetes Obes Metab. 2017;19:1233-1241. doi: 10.1111/dom.12926. PMID:28244632

55. Egan AG, Blind E, Dunder K, et al. Pancreatic safety of incretin-based drugs--FDA and EMA assessment. $N$ Engl J Med. 2014;370: 794-7. doi:10.1056/NEJMp1314078. PMID: 24571751

56. Nauck MA, Meier JJ. Pancreatitis and incretin-based drugs: clarity or confusion? Lancet Diabetes Endocrinol. 2014;2: 92-3. doi:10.1016/S2213-8587(13)70186-4. PMID: 24622699

57. Nauck MA, Meier JJ, Schmidt WE. Incretin-based glucose-lowering medications and the risk of acute pancreatitis and/or pancreatic cancer: Reassuring data from cardio-vascular outcome trials. Diabetes Obes Metab. 2017 Sep;19(9):1327-1328. doi: 10.1111/dom.12981. PMID:28432752 
58. Bragg F, Holmes MV, Iona A, et al. Association between diabetes and cause-specific mortality in rural and urban areas of China. JAMA. 2017;317: 280-289. doi:10.1001/jama.2016.19720. PMID: 28114552

59. Zhao H, Wang L, Wei R, et al. Activation of glucagon-like peptide-1 receptor inhibits tumourigenicity and metastasis of human pancreatic cancer cells via PI3K/Akt pathway. Diabetes Obes Metab. 2014;16: 850-60. doi:10.1111/dom.12291. PMID: 24641303

60. Zhao H, Wei R, Wang L, et al. Activation of glucagon-like peptide-1 receptor inhibits growth and promotes apoptosis of human pancreatic cancer cells in a cAMP-dependent manner. Am J Physiol Endocrinol Metab. 2014;306: E1431-41. doi:10.1152/ajpendo.00017.2014. PMID: 24801389

61. Koehler JA, Kain T, Drucker DJ. Glucagon-like peptide-1 receptor activation inhibits growth and augments apoptosis in murine CT26 colon cancer cells. Endocrinology. 2011;152: 3362-72. doi:10.1210/en.2011-1201. PMID: 21771884

62. Ligumsky H, Wolf I, Israeli S, et al. The peptide-hormone glucagon-like peptide-1 activates cAMP and inhibits growth of breast cancer cells. Breast Cancer Res Treat. 2012;132: 449-61. doi:10.1007/s10549-011-1585-0. PMID: 21638053

63. Shiho K, Takashi N, Chikayo I, et al. GLP-1 action attenuates breast cancer growth and progression. Diabetes Res Clin Pract. 2016;120: S60-S61.doi:10.1016/S0168-8227(16)31048-8

64. Luciani P, Deledda C, Benvenuti S, et al. Exendin-4 induces cell adhesion and differentiation and counteracts the invasive potential of human neuroblastoma cells. PLoS One. 2013;8: e71716.doi:10.1371/journal.pone.0071716. PMID: 23990978

65. Nachnani JS, Bulchandani DG, Nookala A, et al. Biochemical and histological effects of exendin-4 (exenatide) on the rat pancreas. Diabetologia. 2010;53: 153-9. doi:10.1007/s00125-009-1515-4. PMID: 19756486 66. Gier B, Matveyenko AV, Kirakossian D, Dawson D, Dry SM, Butler PC. Chronic GLP-1 receptor activation 
by exendin-4 induces expansion of pancreatic duct glands in rats and accelerates formation of dysplastic lesions and chronic pancreatitis in the Kras(G12D) mouse model. Diabetes. 2012;61: 1250-62. doi:10.2337/db11-1109. PMID: 22266668

67. Singh S, Chang HY, Richards TM, Weiner JP, Clark JM, Segal JB. Glucagon like peptide 1-based therapies and risk of hospitalization for acute pancreatitis in type 2 diabetes mellitus: a population-based matched casecontrol study. JAMA Intern Med. 2013;173: 534-9. doi:10.1001/jamainternmed.2013.2720. PMID: 23440284

68. Raz I, Bhatt DL, Hirshberg B, et al. Incidence of pancreatitis and pancreatic cancer in a randomized controlled multicenter trial (SAVOR-TIMI 53) of the dipeptidyl peptidase-4 inhibitor saxagliptin. Diabetes Care. 2014;37: 2435-41. doi:10.2337/dc13-2546. PMID: 24914244

69. Faillie JL, Azoulay L, Patenaude V, Hillaire-Buys D, Suissa S. Incretin based drugs and risk of acute pancreatitis in patients with type 2 diabetes: cohort study. BMJ. 2014;348: g2780. doi:10.1136/bmj.g2780. PMID: 24764569

70. Giorda CB, Picariello R, Nada E, et al. Incretin therapies and risk of hospital admission for acute pancreatitis in an unselected population of European patients with type 2 diabetes: a case-control study. Lancet Diabetes Endocrinol. 2014;2: 111-5. doi:10.1016/S2213-8587(13)70147-5. PMID: 24622714

71. Li L, Shen J, Bala MM, et al. Incretin treatment and risk of pancreatitis in patients with type 2 diabetes mellitus: systematic review and meta-analysis of randomised and non-randomised studies. BMJ. 2014;348: g2366.doi: 10.1136/bmj.g2366. PMID: 24736555 